\title{
Insomnia and Parental Overprotection are Associated with Academic Stress among Medical Students
}

\author{
Yuree Kang, MD, Changnam Kim, MD, Suyeon Lee, MD, Soyoung Youn, MD \\ Department of Psychiatry, Asan Medical Center, University of Ulsan College of Medicine, Seoul, Korea
}

Background and Objective The purpose of this study was to explore particular aspects of the mental health status of medical students and to identify relationships among them.

Methods All 191 medical students from University of Ulsan College of Medicine were included in this study. Psychological parameters were measured with the Medical Stress Scale (MSS), Insomnia Severity Index, Korean-Parental Overprotection Scale, Patient Health Questionnaire-9 and Academic Motivation Scale.

Results Stressed students (MSS $\geq 28$ ) had significantly higher scores on insomnia severity (5.8 \pm 4.5 vs $4.4 \pm 3.0, \mathrm{p}<0.05)$, depression $(5.7 \pm 4.5$ vs $2.6 \pm 2.4, \mathrm{p}<0.01)$, and amotivation $(9.3 \pm 3.3$ vs $6.9 \pm 2.2, \mathrm{p}<0.01)$ and lower scores of intrinsic motivation $(3.5 \pm 7.1$ vs. $41.7 \pm 7.2, \mathrm{p}<0.01)$ compared to non-stressed students (MSS < 28). Significant correlations were noted between several factors and Medical Stress Scores. Specifically, insomnia, depression, amotivation and maternal 'face culture' of parental overprotection, had independent and significant influences on academic stress reported by medical students $\left(R^{2}=0.39, \mathrm{p}<0.01\right)$.

Conclusions Our findings revealed insomnia, depression, academic motivation and parental overprotection are relevant factors influencing stress in medical students. Current results provide insights for stress management including the importance of parenting intervention.

Sleep Med Res 2017;8(2):92-97

Key Words Insomnia, Medical student, Academic stress, Parental overprotection.

Received: August 16, 2017

Revised: October 2, 2017

Accepted: October 4, 2017

Correspondence

Soyoung Youn, MD

Department of Psychiatry,

Asan Medical Center, University of Ulsan

College of Medicine, 88 Olympic-ro 43-gil,

Songpa-gu, Seoul 05505, Korea

Tel +82-2-3010-1467

Fax +82-2-485-8381

E-mailsoy@amc.seoul.kr

ORCID

Yuree Kang

https://orcid.org/0000-0003-1334-7364

Changnam Kim

https://orcid.org/0000-0001-6767-696X

Suyeon Lee

https://orcid.org/0000-0002-0841-5900

Soyoung Youn

https://orcid.org/0000-0003-4983-4017

\section{INTRODUCTION}

Medical students go through long and intensive academic years in preparation for careers as physicians. There is a growing appreciation of the stress involved in this process. Indeed, previous literature provides hard evidence that a medical school education is highly stressful. One study in England showed that approximately half of the medical students described a stressful incident, the majority of which were related to medical training rather than other problems. ${ }^{1}$

Academic load and emotionally challenging work might be associated with psychiatric morbidities in medical students. Studies from different countries revealed that sleep disturbance among medical students is not only common but also its prevalence is higher than in either non-medical students or the general population. ${ }^{2,3}$ Several studies have also shown that medical students exhibit more symptoms of depression than the general population ${ }^{4,5}$ and depression in first year medical students became twice as prevalent by end of the study period. ${ }^{6}$ In addition, reports by Lloyd and Gartrell ${ }^{5}$ and Vitaliano et al. ${ }^{6}$ demonstrate that medical students had greater mean anxiety levels than the norms for the general population.

Stress levels experienced by each student vary, even though they have the same curriculum and various factors to explain this have been suggested. Prior studies have focused on evaluat- 
ing students' academic stress, psychiatric problems, motivation, personality traits, coping strategies, and more. For instance, depression, ${ }^{7}$ academic motivation ${ }^{8}$ and academic performance ${ }^{9}$ may be related to academic stress, and certain personality traits are associated with motivation ${ }^{10}$ and may predict academic performance. ${ }^{11,12}$ Another study found that maladaptive emotionregulating strategies such as catastrophizing, self-blame, and positive reappraisal contribute to academic stress, and motivation was considered as a mediator. ${ }^{13}$ In our most recent study, high level of depressive symptom was associated with insomnia, amotivation and academic stress in medical student. ${ }^{14}$

Parents in South Korea are renowned for their extraordinary passion toward their children's academic achievement and university entrance. In general, students who achieve academic excellence can enter medical college in South Korea. Therefore, although not applicable to all, medical students in general tend to have grown up with high expectations from their parents since childhood. Previous literature has shown that parental involvements such as overly valuing children's achievements, criticism, or affectionless control can be internalized by children and increase the risks of stress, depression, or anxiety. ${ }^{15-18}$ However to date, there has been no study that conclusively shows a relationship between academic stress experienced by medical students and parental involvement.

With this background, we hypothesized that medical students' academic stress would be associated with their psychological health including sleep and mood. Furthermore, the hypothesis of increased susceptibility to academic stress in students who have parents with overprotective parenting style was investigated. The purpose of this study is to gain greater insights into the relationships between various factors associated with academic stress in medical students, such as insomnia, depression, academic motivation, and parental overprotection. Our findings may form the basis for the management of mental health of Korean medical students.

\section{METHODS}

\section{Participants}

The present study was based on the results of a survey that focused on evaluating mental health status in medical college students. The survey was conducted in spring 2016 at University of Ulsan College of Medicine in Seoul, Korea. We surveyed 201 students at different stages of their education from the second year of pre-medicine through the fourth year of medicine. Aims of the survey were explained in detail and questionnaires were handed out. The students were given ample time to complete the survey. All 201 students finished the study questionnaires and 191 students who gave informed consent to participate were included in the final analysis. This study was approved by the Institutional Review Board (IRB) of Asan Medical Cen- ter, Seoul, Korea (IRB No. 2016-0524).

\section{Measures}

The following questionnaires were used in the present study: Medical Stress Scale (MSS), Insomnia Severity Index (ISI), Patient Health Questionnaire-9 (PHQ-9), Korean-Parental Overprotection Scale (K-POS), and Academic Motivation Scale (AMS).

The MSS consists of eleven items covering five areas and responses were scored using a 5-point Likert scale. ${ }^{19}$ The Korean version modified to reflect cultural differences, was used. ${ }^{20}$ It consists of nine items covering areas including school curriculum/environment, personal competence/endurance, and financial situation. Because a score of 3 ('neither agree nor disagree') for each item yields a total score of 27, a score of 28 was used as the cut-off point for classifying 'stressed' and 'non-stressed' subjects. ${ }^{8}$

The ISI is a 7-item questionnaire that measures the patient's perception of insomnia severity. ${ }^{21}$ Each item is rated on a scale of 0-4 and total score ranges from 0 to 28 , with higher score indicating greater insomnia severity. Scores of 8, 15, and 22 represent subthreshold, moderate and severe insomnia, respectively. The validated Korean version of ISI was used. ${ }^{22}$

The K-POS was used to measure students' perception of parental overprotection. ${ }^{23}$ K-POS was formerly developed to reflect Korean cultural factors. It consists of fifteen items divided into four subscales: goal achieving, face culture, control and identification. Responses are measured on a 5-point Likert scale and higher scores represent higher levels of perceived parental overprotection of each subscale. Tests of convergent validity and construct validity have been demonstrated.

The PHQ-9 is a brief screening and severity measurement tool for major depressive disorder. ${ }^{24}$ It comprises nine items that measure each of the depressive symptoms according to the Diagnostic and Statistical Manual of Mental Disorders-IV diagnostic criteria. The total score ranges from 0 to 27 and scores of $5,10,15$, and 20 represent mild, moderate, moderately severe, and severe depression, respectively. The validated Korean version of PHQ-9 was used. ${ }^{25}$

The AMS was used to assess academic motivation. It consists of a 28-item questionnaire designed to measure three subcategories of intrinsic motivation, extrinsic motivation, and amotivation. ${ }^{26,27}$ Higher scores in each subcategory reflect higher levels of motivation based on that sub-category. A validated Korean version of the AMS was used. ${ }^{20}$

\section{Statistical Analysis}

Descriptive statistics were represented by percentages for qualitative variables, and means and standard deviations for quantitative variables. Chi-square tests and Student's t-test were used to compare the demographic and clinical characteristics of stressed and non-stressed students. The Pearson's correlation was used to identify the association between stress and various 
factors. Multiple regression analysis was performed to determine the best set of predictors for stress. All analyses were conducted using Statistical Package for Social Science (SPSS) version 21.0 (IBM Corp., Armonk, NY, USA), with a significance threshold of $\mathrm{p}<0.05$.

\section{RESULTS}

\section{Demographics and Clinical Characteristics}

Of the 191 students included in this study, 44 (23.0\%) were female. A relatively even distribution throughout academic years was observed in our student sample [second year $(n=41$, $21.4 \%)$ in pre-medicine, first $(\mathrm{n}=33,17.3 \%)$, second $(\mathrm{n}=42$, $22.0 \%)$, third ( $\mathrm{n}=37,19.4 \%)$, and fourth year $(\mathrm{n}=38,19.9 \%)$ in medicine]. The majority of students $(\mathrm{n}=151,79.1 \%)$ did not meet the criteria for insomnia. About one in five had subthreshold insomnia $(\mathrm{n}=36,18.8 \%)$. Few students $(2.1 \%, \mathrm{n}=4)$ scored higher than 15 on the ISI, which suggests the possibility of clini-

Table 1. Demographics and clinical characteristics of stressed and non-stressed medical students

\begin{tabular}{|c|c|c|c|}
\hline \multirow[t]{2}{*}{ Variables } & $\begin{array}{c}\text { Non-stressed } \\
(\mathrm{MSS} \geq 28) \\
(\mathrm{n}=121)\end{array}$ & $\begin{array}{c}\text { Stressed } \\
(\mathrm{MSS}<28) \\
(\mathrm{n}=70)\end{array}$ & \multirow[t]{2}{*}{$\mathrm{p}$} \\
\hline & $\mathrm{n}(\%)$ & $\mathrm{n}(\%)$ & \\
\hline \multirow[t]{2}{*}{ Female gender } & $32(26.9)$ & $12(17.4)$ & 0.13 \\
\hline & Mean \pm SD & Mean \pm SD & \\
\hline School year & $4.1 \pm 1.4$ & $3.7 \pm 1.2$ & 0.09 \\
\hline ISI & $4.4 \pm 3.0$ & $5.8 \pm 4.5$ & $0.02 *$ \\
\hline \multicolumn{4}{|l|}{ K-POS (father) } \\
\hline Goal achieving & $12.5 \pm 3.7$ & $13.1 \pm 3.9$ & 0.32 \\
\hline Face culture & $6.3 \pm 2.2$ & $6.8 \pm 2.7$ & 0.18 \\
\hline Control & $7.4 \pm 3.1$ & $7.3 \pm 3.7$ & 0.79 \\
\hline Identification & $9.4 \pm 2.8$ & $8.8 \pm 2.8$ & 0.21 \\
\hline \multicolumn{4}{|l|}{ K-POS (mother) } \\
\hline Goal achieving & $12.8 \pm 3.4$ & $13.4 \pm 3.3$ & 0.23 \\
\hline Face culture & $6.9 \pm 2.7$ & $7.7 \pm 3.3$ & 0.09 \\
\hline Control & $8.6 \pm 3.7$ & $8.3 \pm 3.7$ & 0.58 \\
\hline Identification & $9.5 \pm 2.7$ & $9.7 \pm 2.0$ & 0.59 \\
\hline PHQ-9 & $2.6 \pm 2.4$ & $5.7 \pm 4.5$ & $<0.01^{\dagger}$ \\
\hline \multicolumn{4}{|l|}{ AMS } \\
\hline Amotivation & $6.9 \pm 2.2$ & $9.3 \pm 3.3$ & $<0.01^{\dagger}$ \\
\hline Intrinsic motivation & $41.7 \pm 7.2$ & $37.5 \pm 7.1$ & $<0.01^{\dagger}$ \\
\hline Extrinsic motivation & $43.5 \pm 5.3$ & $43.2 \pm 6.2$ & 0.71 \\
\hline
\end{tabular}

${ }^{*} \mathrm{p}<0.05 .{ }^{\dagger} \mathrm{p}<0.01$.

MSS: Medical Stress Scale, SD: standard deviation, ISI: Insomnia Severity Index, K-POS: Korean-Parental Overprotection Scale, PHQ-9: Patient Health Questionnaire-9, AMS: Academic Motivation Scale. cally significant insomnia. The mean ISI score was $4.94 \pm 3.70$ (range 0-21). The majority of the students ( $\mathrm{n}=125,65.4 \%$ ) had minimal depression (PHQ-9 score $\leq 4$ ) implying that they were unlikely to need treatment. The incidence of major depression was $7.9 \%(n=15)$ using a PHQ-9 score of 10 as a cut-off. The mean PHQ-9 score was $3.77 \pm 3.69$ (range 0-25).

\section{Group Comparisons According to Academic Stress (Table 1)}

There was no significant difference between stressed (MSS $\geq$ 28 ) and non-stressed students (MSS $<28$ ) in terms of gender. Stressed students had higher scores on the insomnia severity scales compared to non-stressed students (ISI: $5.8 \pm 4.5$ vs 4.4 $\pm 3.0, \mathrm{p}<0.05$ ). There was no significant group difference pertaining to parental overprotection scores. Stressed students had significantly higher scores on the depression scales compared to non-stressed students (PHQ-9: $5.7 \pm 4.5$ vs $2.6 \pm 2.4$, p < 0.01). Among the subcategories of AMS, stressed students showed higher scores in amotivation $(9.3 \pm 3.3$ vs $6.9 \pm 2.2, \mathrm{p}<0.01)$ and lower scores in intrinsic motivation $(37.5 \pm 7.1$ vs $41.7 \pm 7.2$, $\mathrm{p}<0.01)$.

\section{Correlations between Academic Stress and Clinical Variables (Table 2)}

The MSS scores were positively correlated with insomnia severity $(\mathrm{r}=0.32, \mathrm{p}<0.01)$, depression $(\mathrm{r}=0.46, \mathrm{p}<0.01)$, and

Table 2. Correlation of demographic and psychological characteristics with academic stress

\begin{tabular}{lcc}
\hline \multicolumn{1}{c}{ Variables } & MSS (r) & $\mathrm{p}$ \\
\hline School year & -0.14 & $0.04^{*}$ \\
ISI & 0.32 & $<0.01^{\dagger}$ \\
K-POS (father) & & \\
$\quad$ Goal achieving & 0.18 & $0.01^{*}$ \\
Face culture & 0.19 & $<0.01^{\dagger}$ \\
Control & 0.03 & 0.63 \\
Identification & -0.03 & 0.66 \\
K-POS (mother) & & \\
Goal achieving & 0.18 & $0.01^{*}$ \\
Face culture & 0.26 & $<0.01^{\dagger}$ \\
Control & 0.01 & 0.81 \\
Identification & 0.10 & 0.15 \\
PHQ-9 & 0.46 & $<0.01^{\dagger}$ \\
AMS & & $<0.01^{\dagger}$ \\
Amotivation & 0.52 & $<0.01^{\dagger}$ \\
Intrinsic motivation & -0.23 & 0.99 \\
Extrinsic motivation & 0.001 & \\
\hline
\end{tabular}

${ }^{*} \mathrm{p}<0.05 .{ }^{\mathrm{t}} \mathrm{p}<0.01$.

MSS: Medical Stress Scale, ISI: Insomnia Severity Index, K-POS: Korean-Parental Overprotection Scale, PHQ-9: Patient Health Questionnaire-9, AMS: Academic Motivation Scale. 
Table 3. Stepwise linear regression analysis

\begin{tabular}{clcccr}
\hline Outcome variables & Explanatory variables & $\beta$ & $\mathrm{B}$ & $\mathrm{SE}$ & $\mathrm{p}$ \\
\hline MSS $\left(\mathrm{R}^{2}=0.39, \mathrm{p}<0.01\right)$ & ISI & 0.22 & 0.13 & 0.10 & 0.03 \\
& Face culture (mother) & 0.38 & 0.17 & 0.12 & 0.11 \\
& PHQ-9 & 0.37 & 0.22 & 0.01 & $<0.01$ \\
& Amotivation & 0.78 & 0.37 & 0.13 & $<0.01$ \\
\hline
\end{tabular}

SE: standard error, MSS: Medical Stress Scale, ISI: Insomnia Severity Index, PHQ-9: Patient Health Questionnaire-9.

amotivation $(\mathrm{r}=0.52, \mathrm{p}<0.01)$. Among the parental overprotection scores, 'goal achieving' scores (father $\mathrm{r}=0.18, \mathrm{p}=0.01$; mother $\mathrm{r}=0.18, \mathrm{p}=0.01$ ) and 'face culture' scores (father $\mathrm{r}=$ $0.19, \mathrm{p}<0.01$; mother $\mathrm{r}=0.26, \mathrm{p}<0.01$ ) were positively correlated with MSS scores. The MSS scores were negatively correlated with school year $(\mathrm{r}=-0.14, \mathrm{p}=0.04)$ and intrinsic motivation $(\mathrm{r}=-0.23, \mathrm{p}<0.01)$.

\section{Factors Affecting Academic Stress (Table 3)}

We performed multiple regression analysis to construct a best predicting model using variables that showed significance in Pearson's correlation analysis. Insomnia severity scores, maternal 'face culture' subscale scores, depression scale scores, and amotivation were included as independent variables. This model accounted for 39\% of the variation in academic stress levels.

\section{DISCUSSION}

In our study, we found a significant positive association between insomnia and academic stress. Insomnia was a significant variable predicting academic stress in regression analysis. Many studies have indicated that sleep disturbance can either be a comorbidity or a symptom of psychiatric illness. ${ }^{28}$ Evidence shows that insomnia can cause subsequent psychiatric disorders like depression and psychiatric distress. ${ }^{14,29}$ Moreover, good quality sleep is important for optimal neurocognitive and psychomotor performance ${ }^{30}$ and different sleep characteristics influence academic performance. ${ }^{31}$ To optimize the mental health of medical students, future research is needed to explore causative factors underlying sleep problems and develop educational programs to improve sleep in medical students.

Parental overprotection was another associated factor in our results. The finding that 'goal achieving' and 'face culture' score of both parents, respectively, were positively associated with stress level in medical students is interesting and warrants replication. Both subscales were designed as culture-specific constructive domains of Korean parenting when K-POS was originally developed. ${ }^{23}$ It was highlighted earlier that in contrary to 'control' and 'identification' which are culture-general and commonly found in Western studies, 'goal achieving' and 'face culture' are culture-specific factors that reflect more of East Asian values. ${ }^{23}$ One possible explanation is that in East Asian contexts, cultural emphasis is often made to recognize connectedness to close others and to fulfill obligation. ${ }^{32}$ Reflecting this cultural value, identification and control by parents can be often understood as love and support by children while face-concerning or achievement-oriented parents influence more negatively on the stress level of children. This result suggests that it is important to take into account cultural factors in studying student's stress.

Depression was also positively correlated with academic stress and was another strong predicting factor in regression analysis, a finding that is in agreement with our recent studies. ${ }^{13}$ The existence of a bidirectional relationship between stress exposure and development of depression has long been apparent. ${ }^{33,34}$ Our finding also suggests close relatedness between depression and stress level. However, it has to be interpreted cautiously given the cross-sectional design of this study.

Significant correlations between motivation and academic stress were noted in this study. Among types of motivational orientation, amotivation was significantly predictive of stress level according to regression analysis. Such findings are consistent with previous studies which found amotivation was strongly liked to numerous negative outcomes; poor psychosocial adjustment and high levels of perceived stress. ${ }^{13,35}$ Based on selfdetermination theory, ${ }^{36}$ behaviors are non-regulated and nonintentional for amotivated individuals. With no sense of purpose or expectations of changing events, such individuals are likely to experience feelings of incompetence and uncontrollability, a state similar to that of learned helplessness. Intrinsic motivation has been known to enhance quality of learning, academic performance ${ }^{36}$ and general well-being. ${ }^{37}$ In this particular sample of students, we observed negative relationship between intrinsic motivation and stress level, although intrinsic motivation was not significant predictor in regression model.

Our study is limited by several factors. First, our assessments were based on self-reporting. However, we do not regard this as a major limitation because all of the instruments we used have been previously validated. Second, we used cross-sectional design, which precludes determination of whether medical stress is causally related to each variable. Future longitudinal studies are warranted to confirm our results. Third, our study sample was from a single center. Whether this sample is representative of all medical students in South Korea with regard to sex, type of medical school and parenting attitude is unknown. Lastly, because instruments which are not only widely accepted 
but also validated for assessing parental overprotection are scant, K-POS, utilized in the present study, presents its own limitation. The relatively small number of items may have an impact on internal consistency and lack of detectable difference between stressed and non-stressed groups. It is also regrettable that we didn't analyze female and male students separately, since studies have repeatedly suggested that dysfunctional parenting influences recipients in a gender specific manner. ${ }^{38}$ However, to our knowledge, and despite a number of constraints, this study is the first to consider parental overprotection as a relevant measure in contributing to stress levels among medical students.

In sum, our results indicate that academic stress in medical students may be influenced by interaction among various factors including depression, insomnia, motivation and maternal overprotection, especially 'face culture.' Given the implied relevance of these variables on stress levels in medical students, the question of possible intervention becomes important. The importance of intervention particularly with regard to parenting is raised by our study and future investigation is warranted. We believe that our findings will help guide the configuration of stress management in Korean medical students.

\section{Conflicts of Interest}

The authors have no financial conflicts of interest.

\section{Authors' Contribution}

Conceptualization: Kang Y, Youn S. Data curation: Kang Y, Kim C, Lee S. Formal analysis: Kang Y. Investigation: Kang Y, Kim C, Lee S. Methodology: Kang Y, Youn S. Writing_original draft: Kang Y. Writing_review \& editing: Kang Y, Youn S.

\section{REFERENCES}

1. Guthrie EA, Black D, Shaw CM, Hamilton J, Creed FH, Tomenson B. Embarking upon a medical career: psychological morbidity in first year medical students. Med Educ 1995;29:337-41.

2. Veldi M, Aluoja A, Vasar V. Sleep quality and more common sleep-related problems in medical students. Sleep Med 2005;6:269-75.

3. Brick CA, Seely DL, Palermo TM. Association between sleep hygiene and sleep quality in medical students. Behav Sleep Med 2010;8:113-21.

4. Toews JA, Lockyer JM, Dobson DJ, Brownell AK. Stress among residents, medical students, and graduate science $(\mathrm{MSc} / \mathrm{PhD})$ students. Acad Med 1993;68:S46-8.

5. Lloyd C, Gartrell NK. Psychiatric symptoms in medical students. Compr Psychiatry 1984;25:552-65.

6. Vitaliano PP, Maiuro RD, Russo J, Mitchell ES. Medical student distress. A longitudinal study. J Nerv Ment Dis 1989;177:70-6.

7. Ang RP, Huan VS. Relationship between academic stress and suicidal ideation: testing for depression as a mediator using multiple regression. Child Psychiatry Hum Dev 2006;37:133-43.

8. Park J, Chung S, An H, Park S, Lee C, Kim SY, et al. A structural model of stress, motivation, and academic performance in medical students. Psychiatry Investig 2012;9:143-9.

9. Stewart SM, Lam TH, Betson CL, Wong CM, Wong AM. A prospective analysis of stress and academic performance in the first two years of medical school. Med Educ 1999;33:243-50.

10. Tanaka M, Mizuno K, Fukuda S, Tajima S, Watanabe Y. Personality traits associated with intrinsic academic motivation in medical students. Med Educ 2009;43:384-7.

11. Lievens F, Coetsier P, De Fruyt F, De Maeseneer J. Medical students' personality characteristics and academic performance: a five-factor model perspective. Med Educ 2002;36:1050-6.

12. Shen H, Comrey AL. Predicting medical students' academic performances by their cognitive abilities and personality characteristics. Acad Med 1997;72:781-6.

13. Lee S, Youn S, Yi K, Park B, Lee S, An H, et al. Motivation mediates the association between emotional regulation and stress. J Soc Korean Women Psychiatr 2016;14:16-23.

14. Kim C, Lee S, Youn S, Park B, Chung S. Insomnia and neuroticism are related with depressive symptoms of medical students. Sleep Med Res 2017;8:33-8.

15. Horwitz BN, Marceau K, Narusyte J, Ganiban J, Spotts EL, Reiss D, et al. Parental criticism is an environmental influence on adolescent somatic symptoms. J Fam Psychol 2015;29:283-9.

16. Cheung CS, Pomerantz EM. Value development underlies the benefits of parents' involvement in children's learning: A longitudinal investigation in the United States and China. J Educ Psychol 2015;107:309-20.

17. Goschin S, Briggs J, Blanco-Lutzen S, Cohen LJ, Galynker I. Parental affectionless control and suicidality. J Affect Disord 2013;151:1-6.

18. Otani K, Suzuki A, Matsumoto Y, Sadahiro R, Enokido M. Affectionless control by the same-sex parents increases dysfunctional attitudes about achievement. Compr Psychiatry 2014;55:1411-4.

19. Vitaliano PP, Maiuro RD, Mitchell E, Russo J. Perceived stress in medical school: resistors, persistors, adaptors and maladaptors. Soc Sci Med 1989;28:1321-9.

20. Ahn D, Park G, Baek KJ, Chung SI. Academic motivation, academic stress, and perceptions of academic performance in medical students. Korean J Med Educ 2007;19:59-71.

21. Bastien CH, Vallières A, Morin CM. Validation of the Insomnia Severity Index as an outcome measure for insomnia research. Sleep Med 2001;2:297-307.

22. Cho YW, Song ML, Morin CM. Validation of a Korean version of the insomnia severity index. J Clin Neurol 2014;10:210-5.

23. Chung KM, Yoon JL. Development and validation of the Korean-Parental Overprotection Scale: For high school students. Kor J School Psychol 2015;12:1-26.

24. Kroenke K, Spitzer RL, Williams JB. The PHQ-9: validity of a brief depression severity measure. J Gen Intern Med 2001;16:606-13.

25. An JY, Seo ER, Lim KH, Shin JH, Kim JB. Standardization of the Korean version of screening tool for depression (Patient Health Questionnaire-9, PHQ-9). J Korean Soc Biol Ther Psychiatry 2013;19:47-56.

26. Fairchild AJ, Horst SJ, Finney SJ, Barron KE. Evaluating existing and new validity evidence for the Academic Motivation Scale. Contemp Educ Psychol 2005;30:331-58.

27. Vallerand RJ, Pelletier LG, Blais MR, Briere NM, Senecal C, Vallieres EF. The Academic Motivation Scale: A Measure of Intrinsic, Extrinsic, and Amotivation in Education. Educ Psychol Meas 1992;52:1003-17.

28. Ford DE, Kamerow DB. Epidemiologic study of sleep disturbances and psychiatric disorders. An opportunity for prevention? JAMA 1989;262: 1479-84.

29. Chang PP, Ford DE, Mead LA, Cooper-Patrick L, Klag MJ. Insomnia in young men and subsequent depression. The Johns Hopkins Precursors Study. Am J Epidemiol 1997;146:105-14.

30. Giri P, Baviskar M, Phalke D. Study of sleep habits and sleep problems among medical students of pravara institute of medical sciences loni, Western maharashtra, India. Ann Med Health Sci Res 2013;3:51-4.

31. Gomes AA, Tavares J, de Azevedo MH. Sleep and academic performance in undergraduates: a multi-measure, multi-predictor approach. Chronobiol Int 2011;28:786-801.

32. Fu AS, Markus HR. My mother and me: Why tiger mothers motivate Asian Americans but not European Americans. Pers Soc Psychol Bull 2014;40:739-49.

33. Kendler KS, Karkowski LM, Prescott CA. Causal relationship between stressful life events and the onset of major depression. Am J Psychiatry 1999;156:837-41. 
34. Caspi A, Sugden K, Moffitt TE, Taylor A, Craig IW, Harrington H, et al. Influence of life stress on depression: moderation by a polymorphism in the 5-HTT gene. Science 2003;301:386-9.

35. Baker SR. Intrinsic, extrinsic, and amotivational orientations: Their role in university adjustment, stress, well-being, and subsequent academic performance. Current Psychology 2004;23:189-202.

36. Deci EL, Ryan RM. Intrinsic motivation and self-determination in human behavior. New York: Springer Science \& Business Media 1985;24570.
37. Ryan RM, Deci EL, Grolnick WS, La Guardia JG. The significance of autonomy and autonomy support in psychological development and psychopathology. In: Cicchetti D, Cohen DJ. Developmental psychopathology. Vol. 1: Theory and methods. 2nd ed. Oxford: John Wiley \& Sons, Inc. 2006;795-849.

38. Otani K, Suzuki A, Matsumoto Y, Shibuya N, Sadahiro R, Enokido M. Parental overprotection engenders dysfunctional attitudes about achievement and dependency in a gender-specific manner. BMC Psychiatry 2013;13:345. 